\title{
Cystatin-C as a Marker for Renal Impairment in Preeclampsia
}

\author{
Apeksha Niraula, ${ }_{1}^{1}$ Madhab Lamsal, ${ }^{1}$ Nirmal Baral, ${ }^{1}$ Shankar Majhi, ${ }^{2}$ Seraj Ahmed Khan, \\ Pritha Basnet, ${ }^{3}$ and Kashyap Dahal ${ }^{4}$
}

${ }^{1}$ Department of Biochemistry, B.P. Koirala Institute of Health Sciences, Dharan, Nepal

${ }^{2}$ Department of Biochemistry, School of Medicine, Xavier University, Oranjestad, Aruba

${ }^{3}$ Department of Obstetrics and Gynecology, B.P. Koirala Institute of Health Sciences, Dharan, Nepal

${ }^{4}$ Department of Internal Medicine, B.P. Koirala Institute of Health Sciences, Dharan, Nepal

Correspondence should be addressed to Apeksha Niraula; apekshyaniraula@gmail.com

Received 22 March 2017; Revised 4 June 2017; Accepted 7 June 2017; Published 11 July 2017

Academic Editor: Kasper Karmark Iversen

\begin{abstract}
Copyright ( 2017 Apeksha Niraula et al. This is an open access article distributed under the Creative Commons Attribution License, which permits unrestricted use, distribution, and reproduction in any medium, provided the original work is properly cited.

Preeclampsia is a devastating pregnancy-associated disorder characterized by the onset of hypertension, proteinuria, and edema with limited plausible pathophysiology known. Cystatin-C, a novel marker for the detection of renal impairment, is increased in preeclampsia at an early stage. This study was aimed to evaluate the diagnostic efficiency of Cystatin-C as an early marker of renal function in preeclampsia comparing it to the traditional renal markers. A hospital based comparative cross-sectional study was performed on 104 women (52 diagnosed cases of preeclampsia and 52 healthy pregnant women). Concentrations of Cystatin-C, creatinine, urea, and uric acid were measured in both the study groups. Mean serum Cystatin-C and uric acid levels were elevated in preeclampsia cases compared to controls ( $1.15 \pm 0.37$ versus $0.55 \pm 0.12 ; 5.40 \pm 1.44$ versus $3.97 \pm 0.68$, resp.). ROC curve depicted that Cystatin-C had the highest diagnostic efficiency (sensitivity, 88.24\%; specificity, 98.04\%) compared to creatinine and uric acid. Serum Cystatin-C consequently seemed to closely reflect the renal functional changes, which are believed to lead to increased blood pressure levels and urinary excretion of albumin and may thus function as a marker for the stage of the transition between normal adaptive renal changes at term and preeclampsia.
\end{abstract}

\section{Introduction}

Hypertension is one of the common medical complications of pregnancy and contributes significantly to maternal and perinatal morbidity and mortality [1]. Hypertensive disorders in pregnancy are responsible for 76,000 maternal and 500,000 infant deaths each year worldwide. A World Health Organization (WHO) analysis of maternal deaths reveals that hypertensive disorders are responsible for $16.1 \%$ maternal deaths in developed countries and is a major contributor to maternal death in Africa (9.1\%) and Asia (9.1\%) [2]. Preeclampsia (PE), a multisystem disorder of unknown etiology, is characterized by development of hypertension to the extent of $140 / 90 \mathrm{mmHg}$ or more with proteinuria after the 20th week in a previously normotensive and nonproteinuric woman with proteinuria [1]. It is defined as maternal systolic blood pressure $>140 \mathrm{mmHg}$ and/or diastolic blood pressure > $90 \mathrm{mmHg}$ measured on two occasions separated by at least
6 hours and proteinuria $>300 \mathrm{mg}$ in a 24-hour period or qualitative, $>1+$, after 20 weeks of gestation following the guidelines of the American College of Obstetricians and Gynecologists (ACOG), 2002.

The kidneys play an essential role, both in the adaptive physiology of normal pregnancy and in the pathophysiology of PE [3] and some changes in renal function are found to be common to term pregnancy and PE [4]. But, the challenge to every clinician in the present context is to diagnose the renal impairment at an early stage to prevent this leading cause of fetal morbidity and mortality to progress into a severe stage (eclampsia) [5]. Efforts to find an effective predictive test early in pregnancy have not been successful in a lowrisk population and there is no gold standard diagnostic test to define PE [6]. The condition is a multisystem disorder, where different aspects of the disease are used in different classifications of hypertensive disorders in pregnancy. This 
makes it difficult to establish a clear-cut population of women at risk or women with developed PE for investigation and also confuses interpretation of the literature in the field where separate classifications are used [7]. The only consistently found pathological lesion in $\mathrm{PE}$ is the renal lesion termed glomerular endotheliosis, which has been regarded as pathognomic for the condition. $\mathrm{PE}$, which is characterized by widespread maternal endothelial dysfunction, inevitably may compromise glomerular dynamics and barrier function [4]. Assessment of renal function is, therefore, important in the evaluation of the pregnant patient with hypertension.

Creatinine is the most widely used biomarker of kidney function but is impervious in the early stages of renal impairment [8]. Serum creatinine levels are elevated in patients with renal malfunction especially with the significant decrease in glomerular filtration. Vasodilation of the renal vessels in pregnancy causes $50-80 \%$ increase in plasma flow and change in glomerular filtration rate (GFR), which further complicates the use of serum creatinine as a marker of GFR in pregnancy [9]. Uric acid (UA) is filtered, reabsorbed, and secreted by the kidney. Hypovolemia, an early change in PE, increases UA reabsorption which could increase serum UA concentrations. However, increased UA precedes the reduction in plasma volume [10]. Increased UA production from maternal, fetal, or placental tissues through increased tissues breakdown and/or increased xanthine oxidase activity may also be the cause of increased concentration [11]. Uric acid is also a predictive marker of eclampsia and fetal outcome [12]. Uric acid was popularly used as a marker of GFR when monitoring renal function in PE. Its serum concentration increased with the severity of PE and was assigned as a good predictor in clinical observation, even a pathogenic factor in the pathophysiology of PE. But results of some investigations made UA assessment in the estimation of hypertensive disorder of pregnancy fall into disfavor [13].

All these explanations suggest that these traditional markers of renal function are unable to assess the renal impairment at an early stage and also to detect the reduced GFR in early stages of kidney dysfunction; hence search for new biomarker like Cystatin- $\mathrm{C}$ is suggested. In order to overcome this hindrance in estimating renal function in pregnant women, studies have demonstrated that serum Cys- $\mathrm{C}$ can reliably reflect the GFR in both healthy and hypertensive pregnant women [13].

Therefore, this study was undertaken to see the diagnostic efficacy of serum Cys-C as a marker of early renal impairment in $\mathrm{PE}$ and compare it with other traditional renal markers.

\section{Aims and Objectives}

(i) To compare serum Cystatin-C, creatinine, and uric acid levels in PE and normal pregnant women.

(ii) To evaluate the diagnostic value of serum Cystatin$\mathrm{C}$ level as an alternative marker of renal function in preeclampsia.

\section{Methods}

The study was conducted at the Department of Obstetrics and Gynecology in collaboration of Department of Biochemistry,
BPKIHS, Dharan, Nepal, after being approved by the Institutional Ethical Review Board (IERB), BPKIHS. An informed consent was obtained from all the study participants. Renal function was investigated in two groups of pregnant women: one with preeclampsia $(n=51)$ and the other of healthy pregnant women $(n=51)$.

Blood pressure measurement and urine analysis were performed at the beginning of the pregnancy to exclude preexisting proteinuria or renal disease. Maternal conditions potentially affecting GFR during the study (pregestational hypertension, diabetes, and other concomitant renal diseases), if present, were not included.

The common inclusion criteria for both groups were normal fetal morphology and the absence of concomitant disease and gestation between $\geq 24$ and 36 gestational weeks. Additional inclusion criteria for preeclampsia were a systolic blood pressure level of $140 \mathrm{mmHg}$ or higher or a diastolic blood pressure level of $90 \mathrm{mmHg}$ or higher that occurred after 20 weeks of gestation complicated with proteinuria, defined as the presence of $0.3 \mathrm{~g}$ or more of protein in a $24 \mathrm{~h}$ urine specimen. Other parameters included in the study were gestational age, parity, and body mass index (BMI). Systolic blood pressure (SBP), diastolic blood pressure (DBP), and mean arterial pressure (MAP) were also noted in both the study groups.

The results from PE group were compared with that of the healthy age and gestational week matched control group.

Serum Cystatin-C levels were measured by Nephelometry method using a Fully Automated Autoanalyser (Accent 2000) with intra- and interassay \% CV less than 5\% and according to the procedure recommended by reagent manufacturer. Serum creatinine levels were measured by Jaffe's method, in cobas C311 Autoanalyser (Roche Diagnostics) with intra- and interassay \% CV less than $2.45 \%$ and according to the procedure recommended by the manufacturer. Serum uric acid levels were measured by standardized enzymatic PAP method with uricase and peroxidase, in cobas C311 Autoanalyser with intra- and interassay \% CV less than $2.44 \%$ and according to the procedure recommended by the manufacturer. Serum urea was estimated by standard urease method in cobas C311 Autoanalyser with intra- and interassay \% CV less than $2.44 \%$ and according to the procedure recommended by the manufacturer. In patients with $\mathrm{PE}$, total urinary protein levels were measured by modification of the dye binding method used by Fujita et al. [14] and with commercial uric 3V SGO 3100 .

\section{Statistical Analysis}

Data were initially checked for normal distribution by Kolmogorov-Smirnov test. For normally distributed data, mean with SD, Pearson's correlation, and Student's $t$-tests were applied. If data was not normally distributed, nonparametric tests were applied. Independent Student's $t$-test was used to compare Cys-C levels, creatinine, urea, and uric acid between the two groups. Pearson's correlation was used to correlate Cys-C levels with POG, BMI, SBP, DBP, MAP, urea, and uric acid in $\mathrm{PE}$ and control groups, respectively. 
TABLE 1: Baseline and clinical parameters of study participants.

\begin{tabular}{lccc}
\hline General characteristics & PE $(n=51)$ & Control $(n=51)$ & $p$ value \\
\hline Age (years) & $26.84 \pm 5.20$ & $25.84 \pm 4.54$ & $0.304^{\mathrm{a}}$ \\
POG (weeks) & $35.02 \pm 4.59$ & $29.35 \pm 3.35$ & $0.001^{\text {a* }}$ \\
BMI (Kg/m $\left.{ }^{2}\right)$ & $28.71 \pm 4.30$ & $24.47 \pm 2.86$ & $0.001^{\text {a* }}$ \\
SBP (mmHg) & $146.86 \pm 10.67$ & $104.31 \pm 10.24$ & $0.001^{\text {a* }}$ \\
DBP (mmHg) & $95.69 \pm 8.77$ & $71.76 \pm 7.67$ & $0.001^{\text {a* }}$ \\
MAP & $112.74 \pm 8.21$ & $8.21 \pm 7.86$ & $0.001^{\text {a }^{\text {a }}}$ \\
\hline
\end{tabular}

${ }^{a}$ Independent $t$-test; ${ }^{*} p$ value $<0.05$ is considered to be significant.

TABLE 2: Biochemical parameters in preeclampsia and control group.

\begin{tabular}{lccc}
\hline Biochemical parameters & PE $(n=51)$ & Control $(n=51)$ & $p$ value \\
\hline Cystatin-C $(\mathrm{mg} / \mathrm{L})$ & $1.15 \pm 0.37$ & $0.55 \pm 0.12$ & $0.001^{\mathrm{a} *}$ \\
Creatinine $(\mathrm{mg} / \mathrm{dl})$ & $0.4(0.3,0.5)$ & $0.5(0.35,0.57)$ & $0.178^{\mathrm{b}}$ \\
Urea $(\mathrm{mg} / \mathrm{dl})$ & $15.60 \pm 6.10$ & $15.91 \pm 5.01$ & $0.783^{\mathrm{a}}$ \\
Uric acid $(\mathrm{mg} / \mathrm{dl})$ & $5.40 \pm 1.44$ & $3.97 \pm 0.68$ & $0.001^{\mathrm{a} *}$ \\
\hline
\end{tabular}

${ }^{\mathrm{a}}$ Independent $t$-test; ${ }^{\mathrm{b}}$ Mann-Whitney $U$ test $\left(0.4\right.$ is the median value and 0.3 and 0.5 represent the 25 th and 75 th percentile); ${ }^{*} p$ value $<0.05$ is considered to be statistically significant.

TABLE 3: Multiple linear regression analysis of DBP with the ANOVA table for the model.

\begin{tabular}{|c|c|c|c|c|}
\hline Variables & Coefficient & SE & $t$-value & $p$ value \\
\hline Intercept & 114.55 & 15.65 & - & - \\
\hline Age (years) & -0.208 & 0.235 & 1.488 & 0.144 \\
\hline POG (weeks) & -0.017 & 0.289 & -0.114 & 0.910 \\
\hline BMI $\left(\mathrm{kg} / \mathrm{m}^{2}\right)$ & -0.420 & 0.271 & -3.166 & $0.003^{*}$ \\
\hline Cystatin-C (mg/L) & 0.214 & 0.271 & 1.434 & 0.159 \\
\hline Creatinine (mg/dl) & 0.119 & 2.132 & 0.904 & 0.371 \\
\hline Urea (mg/dl) & 0.121 & 0.189 & 0.922 & 0.362 \\
\hline Uric acid (mg/dl) & 0.207 & 0.851 & 1.475 & 0.148 \\
\hline
\end{tabular}

Coefficient: regression coefficient; SE: standard error. ${ }^{*} p$ value $<0.05$ is considered to be statistically significant.

Spearman's correlation was used to correlate creatinine and Cys-C levels in PE and control groups, respectively. Multiple linear regression was also performed to explore the association between the predictors, namely, age, POG, BMI, Cys-C, creatinine, urea, and UA and DBP in PE. ROC curve was used to evaluate the diagnostic utility of $\mathrm{Cys}-\mathrm{C}$, creatinine, and UA as a marker of renal function in PE.

\section{Results}

Descriptive characteristics of the study participants are illustrated in Table 1. Overall, most of the study participants in both the study groups were multigravida and nullipara. Our study shows that the female developed PE in late gestational period $(26.84 \pm 5.20)$. The details of the baseline and clinical parameters have been summarized in Table 1 . The mean gestational age of pregnant women with PE was 35.02 \pm 4.59 . BMI was higher in preeclamptic patients than in control group $(28.71 \pm 4.30$ versus $24.47 \pm 2.86)$. Mean SBP and DBP $(\mathrm{mm}$ of $\mathrm{Hg})$ were higher in preeclamptic patients than in control group $(146.36 \pm 10.67 ; 112.74 \pm 8.21$ and $104.31 \pm 10.24$; $71.76 \pm 7.67$, resp.). MAP was significantly higher in PE (112.74 $\pm 8.21)$ than in control group $(82.61 \pm 7.86)$. The details of the baseline and clinical parameters have been summarized in Table 1.

Mean serum Cystatin-C level was higher in PE compared to the control group $(1.15 \pm 0.37$ versus $0.55 \pm 0.12)$ and was statistically significant. Median serum creatinine was higher in PE compared to control group but the distribution between the two groups was statistically insignificant: $0.40(0.30,0.50)$ versus $0.50(0.35,0.57)$. Serum urea was slightly lower in $\mathrm{PE}$ compared to control group $(15.60 \pm 6.10$ versus $15.91 \pm$ 5.01) without any statistical significance. Serum uric acid was significantly higher in preeclamptic patients compared to control group; values are $5.40 \pm 1.44$ versus $3.97 \pm$ 0.68 , respectively, as depicted in Table 2 . Multiple regression analysis showed that the predictors significantly predict the outcome of DBP and only BMI remained as a single predictor significantly influencing the DBP as illustrated in the table as shown in Table 3. Levels of Cystatin-C have been shown to be unaffected in various age groups. The results of the present study found similar findings; that is, the concentration of serum Cystatin- $\mathrm{C}$ was unchanged between various age but the difference was not significant (Table 4). 
TABLE 4: Comparison of Cystatin-C with various age groups in PE and control group.

\begin{tabular}{|c|c|c|c|c|c|}
\hline Variables & $1(<20$ yrs $)$ & $2(20-24$ yrs $)$ & 3 (25-30 yrs) & $4(>30$ yrs $)$ & $p$ value \\
\hline \multicolumn{6}{|c|}{ Cystatin-C (mg/L) } \\
\hline $\mathrm{PE}$ & $1.10 \pm 0.14$ & $1.24 \pm 0.39$ & $1.01 \pm 0.15$ & $1.23 \pm 0.49$ & 0.292 \\
\hline Control group & $0.58 \pm 0.10$ & $0.55 \pm 0.11$ & $0.55 \pm 0.11$ & $0.54 \pm 0.18$ & 0.978 \\
\hline
\end{tabular}

TABLE 5: Showing sensitivity and specificity of various renal markers.

\begin{tabular}{|c|c|c|c|c|c|c|c|}
\hline Parameters & AUC (95\% CI) & Sensitivity & Specificity & $+\mathrm{LR}$ & $-\mathrm{LR}$ & PPV & NPV \\
\hline Cystatin-C & 0.993 & $88.24 \%$ & $98.04 \%$ & $\begin{array}{c}45.0 \\
(6.4-314.17)\end{array}$ & $\begin{array}{c}0.12 \\
(0.06-0.25)\end{array}$ & $97.83 \%$ & $89.29 \%$ \\
\hline Creatinine & 0.423 & $62.75 \%$ & $27.45 \%$ & $\begin{array}{c}0.86 \\
(0.66-1.13)\end{array}$ & $\begin{array}{c}1.36 \\
(0.77-2.40)\end{array}$ & $46.38 \%$ & $42.42 \%$ \\
\hline Uric acid & 0.815 & $79.07 \%$ & $71.19 \%$ & $\begin{array}{c}3.78 \\
(2.02-7.05)\end{array}$ & $\begin{array}{c}0.40 \\
(0.27-0.61)\end{array}$ & $66.67 \%$ & $82.35 \%$ \\
\hline
\end{tabular}

There was a significant positive correlation between Cystatin-C level and uric acid $(r=0.33$; $p$ value $<0.05)$. There was also a significant negative correlation between eGFR and serum Cystatin-C level $(r=-0.777, p<0.001)$. The negative correlation implies that as the eGFR level decreases with the progression of kidney disease, there is a subsequent rise in serum Cystatin-C level.

eGFR as calculated by CKD-EPI equation by serum Cystatin- C level (according to KDIGO 2012) was significantly reduced in $\mathrm{PE}$ as compared to control group. Mean eGFR in PE was $85.86 \pm 28.53$ and $130.58 \pm 14.22$ in control group with the difference being statistically significant. The details have been illustrated in Table 4. Receptor operating characteristics (ROC) curve was used to determine the diagnostic efficacy of three markers, namely, Cys-C, creatinine, and uric acid in PE. Cystatin-C showed the superior diagnostic accuracy compared to the other two traditional renal markers. From the curve, the cut-off value for Cystatin$\mathrm{C}$ was determined with maximum sensitivity and specificity to be 0.9 . Similarly, for creatinine, the cut-off was taken as 0.38 and for UA 4.25, respectively. ROC curve has been shown in Figure 1 and sensitivity and specificity of all the three markers have been illustrated (Table 5).

\section{Discussion}

Renal impairment in PE has been implicated to various reasons, the most likely being hemodynamic changes [15] and glomerular endotheliosis [16], as well as podocyte damage [17]. Our study showed that serum Cystatin-C is significantly higher in PE compared to control group. This finding was in accordance with $[15,18,19]$. Preeclamptic patients are at increased risk of renal impairment, though the dysfunction is usually undershadowed during the gestational period. The patients who developed $\mathrm{PE}$ are also at increased risk of developing PE in their subsequent pregnancies [20, 21]. The renal impairment if undiagnosed early can progress to renal failure and also lead to other vascular disorders later in life [22-24]. Mean BMI in PE was higher than control group (refer to Table 1). BMI is one among the three risk factors for increased SBP and DBP, the other two being increased maternal age and gestational age. Multiple regression analysis showed that the predictors significantly predict the outcome of DBP and only BMI remained as a single predictor which

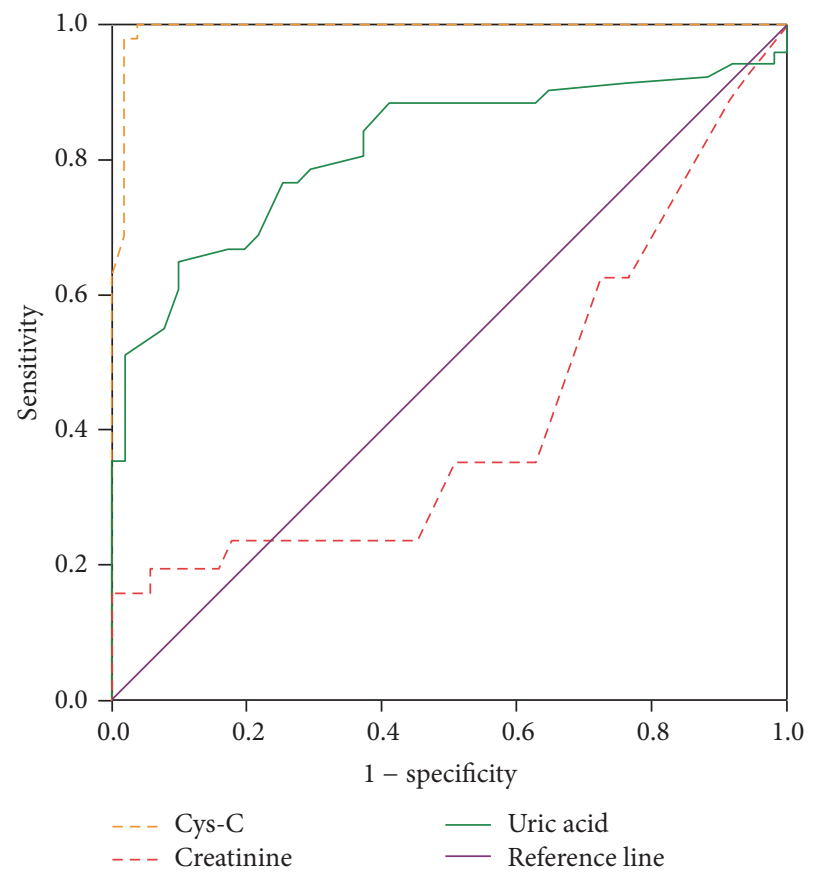

FIGURE 1: ROC curve to determine the diagnostic utility of Cystatin$C$, creatinine, and uric acid in PE. Cystatin-C showed the superior diagnostic accuracy compared to the other two traditional renal markers. From the curve, we determined the cut-off value for Cystatin-C with maximum sensitivity and specificity to be 0.9 . Similarly, for creatinine, the cut-off was taken as 0.38 and for uric acid 4.25 , respectively.

significantly influenced the DBP as depicted in Table 3. Several investigations have indicated that serum Cystatin$\mathrm{C}$ is a better marker for GFR than serum creatinine, in particular for individuals with small to moderate decreases in GFR [25]; only few investigations regarding Cystatin-C levels in pregnancy have been published $[18,26,27]$. Though, 
it would have been logical to expect a decreased value of serum Cystatin-C as the renal plasma flow increases during pregnancy leading to about $40 \%$ higher GFR, determined as the plasma clearance rate of the low molecular mass substance Iohexol [28]. To this, numerous explanations have been put forward for the fact that serum Cystatin-C does not decrease during pregnancy.

Production of Cystatin-C might be increased during pregnancy due to an increased number of nucleated cells which is supported by a study showing serum Cystatin-C is increased during twin pregnancy $[18,25]$. It is more likely that the increase in Cystatin-C levels in pregnancy is due to an altered filtration process than to an increased production rate, as the serum levels of Cystatin-C were not found to correlate to fetal or placental weight in a study conducted by [29] suggesting that Cystatin-C does not cross the placental barrier. Thus, we speculate that there could be a shift towards a more cationic glomerular barrier in pregnant women, resulting in higher serum concentrations of Cystatin-C during pregnancy. Hence, it would be necessary to monitor kidney function during pregnancy by serum Cystatin-C for detection of abnormal kidney function, as serum Cystatin-C seems to reflect an altered filtration process in pregnancy at an early period serum. Cystatin- $\mathrm{C}$ is considered to be a superior marker for assessment of renal function and GFR (refer to Table 2) more closely than traditional renal markers, namely, creatinine and uric acid, as Cystatin-C level is not affected by age, gender, race, ethnicity, muscle mass, and diet [30,31]. The present study analyzed if the level of Cystatin-C varied among the age groups in PE and control group. We did not find any rise in Cystatin-C levels in higher age group, though the difference between the two groups was not statistically significant as shown in Table 4.

As demonstrated by ROC, analysis of the data in the present study is depicted in Figure 1 and Table 5. Serum Cystatin-C showed a highest diagnostic accuracy compared to serum creatinine and serum uric acid $[18,26,32]$. Serum uric acid showed a higher diagnostic accuracy than creatinine and has also been shown to be a useful predictor of fetal outcome in preeclampsia [33-36], though increasing serum levels in PE reflects an enhanced reabsorption in the proximal tubules and not a reduced Cystatin-C [37]. This finding was in accordance with the study done by [18], in which they found that Cystatin-C was higher among all of the PE patients than the control group. Serum creatinine is also of limited use in the assessment of the GFR, which can be reduced by $50 \%$ without causing abnormal serum creatinine concentrations [38-40]. Several of the patients with the most severe preeclampsia had normal creatinine levels in our study, whereas all patients with severe preeclampsia had Cystatin$\mathrm{C}$ levels raised above the upper reference limit for normal term pregnancy. Preeclampsia can be diagnosed easily by determining hypertension with proteinuria, but the diagnosis of the true condition associated with the increased risk can still be elusive, as pregnant women can present with hypertension and proteinuria due to other conditions as well, and a preeclamptic state can be present without raised blood pressure or albuminuria [41]. Moreover, blood pressure levels and proteinuria are unstable markers, often varying within a wide range during the course of the disease [42]. The estimation of serum Cystatin-C could be helpful in the diagnosis of PE, reflecting a different feature of the disease as a stable indicator of an altered filtration process and may also prove valuable for the monitoring of GFR in renal disease in pregnancy and in PE [43] (Table 2).

\section{Conclusion}

Preeclampsia is still a leading cause of maternal morbidity and mortality in developed and underdeveloped countries like ours. Renal dysfunction plays a central and initial role in pathophysiology of PE. Hence, assessment of renal function plays a vital role in monitoring and prediction of severity in PE. Thus, an early marker of renal impairment is needed in the diagnosis and thereby preventing progression of $\mathrm{PE}$ to eclampsia.

\section{Ethical Approval}

This study has been approved by the Institutional Ethical Review Board, B.P. Koirala Institute of Health Sciences, Dharan, Nepal. The date of approval was 9 June 2014 and the code is IERB/267/014.

\section{Disclosure}

Madhab Lamsal, Nirmal Baral, Shankar Majhi, Seraj Ahmed Khan, Pritha Basnet, and Pritha Dahal are coauthors. No funding was done for the study from any organization. The investigator herself had contributed for the purchase of reagents.

\section{Conflicts of Interest}

There are no conflicts of interest regarding the publication of this study.

\section{Authors' Contributions}

The principal investigator is Dr. Apeksha Niraula, M.D. biochemistry, BPKIHS. This study is a part of her M.D. dissertation. She has designed the study, collected the history and blood samples from the study participants after the inclusion criteria have been met, and done the required laboratory parameters on the blood samples on her own. The measurement of the analyte, statistical analysis of the data, and writing of the paper have also been completed by the principal author. The coinvestigator, Professor Madhab Lamsal, Ph.D. biochemistry, BPKIHS, is the guide of the principal author during her postgraduate study. He has contributed a lot as a supervisor and mentor to the author by helping in the design of the study and during writing of the article. The coinvestigator, Professor Nirmal Baral, M.D. biochemistry, BPKIHS, is also one of the mentors of the author. He has contributed for helping to conduct the study more conveniently and has also aided in editing of the article. The coinvestigator, Professor Shankar Majhi, M.D. 
biochemistry, BPKIHS, is also one of the mentors of the author. He has contributed during the implementation of the idea to conducting the study. He has also helped during the analysis of the analyte. The coinvestigator, Dr. Seraj Ahmed Khan, M.D. biochemistry, BPKIHS, has contributed by the implementation of the idea by designing the study. He has been actively involved in statistical analysis and report writing of the study. The coinvestigator, Dr. Pritha Basnet, M.D. obstetrics and gynecology, BPKIHS, has actively contributed during the sample collection and has been a great help for statistical analysis. The coinvestigator, Dr. Kashyap Dahal, M.D. internal medicine, BPKIHS, has helped during the sample collection for preeclampsia by thorough clinical examination to exclude the patients who do not meet the inclusion criteria for the study. He has also contributed his valuable time in interpretation of the statistical results and during writing up the article. All of the authors mentioned above are genuine and have helped equally for the completion of the study.

\section{Acknowledgments}

Apeksha Niraula would like to acknowledge her parent institute B.P. Koirala Institute of Health Sciences, Dharan, Nepal, for providing the platform to conduct the study. She also expresses her sincere gratitude to the study participants without whom the study would have been impossible. The author also acknowledges the 15th Asian Pacific Congress of Nephrology \& 52nd Australian and New Zealand Society of Nephrology ASM held at Perth, Western Australia, for providing an opportunity and travel grant to attend and do the oral presentation of the paper.

\section{References}

[1] D. Dutta and H. C. Konar, "Hypertensive disorders in pregnancy," in DC Dutta's Textbook of Obstetrics, pp. 219-240, 2015.

[2] K. S. Khan, D. Wojdyla, L. Say, A. M. Gülmezoglu, and P. F. van Look, "WHO analysis of causes of maternal death: a systematic review," The Lancet, vol. 367, no. 9516, pp. 1066-1074, 2006.

[3] L. C. Chesley, "Diagnosis of preeclampsia," Obstetrics \& Gynecology, vol. 65, pp. 423-425, 1985.

[4] C. W. G. Redman, G. P. Sacks, and I. L. Sargent, "Preeclampsia: an excessive maternal inflammatory response to pregnancy," American Journal of Obstetrics \& Gynecology, vol. 180, no. 2, pp. 499-506, 1999.

[5] K. H. Coppage and B. M. Sibai, "Preeclampsia and Eclampsia," in Gynecology and Obstetrics, p. 291, Lippincott William \& Wilkins, 2004.

[6] R. Friedman, M. J. De Azevedo, and J. L. Gross, "Is endogenous creatinine clearance still a reliable index of glomerular filtration rate in diabetic patients?" Braz J Med Biol Res, vol. 21, 941, no. 5, p. $944,1988$.

[7] M. A. Brown and M. L. Buddle, "What's in a name? Problems with the classification of hypertension in pregnancy," Journal of Hypertension, vol. 15, no. 10, pp. 1049-1054, 1997.

[8] E. Eiland, C. Nzerue, and M. Faulkner, "Preeclampsia 2012," Journal of Pregnancy, vol. 2012, Article ID 586578, 7 pages, 2012.
[9] K. Sumithra, C. Vibha, and H. L. Vishwanath, "Study of Serum cystatin C in Preeclampsia," Int J Curr Res, vol. 5, no. 10, pp. 2994-2996, 2013.

[10] S. A. Bainbridge and J. M. Roberts, "Uric acid as a pathogenic factor in Preeclampsia," Placenta, vol. 29, pp. 67-72, 2008.

[11] E. D. M. Gallery, S. N. Hunyor, and A. Z. Györy, "Plasma volume contraction: A significant factor in both pregnancy-associated hypertension (preeclampsia) and chronic hypertension in pregnancy," QJM, vol. 48, no. 4, pp. 593-602, 1979.

[12] E. J. Lamb and C. P. Price, "Kidney Function Tests," in Teitz Textbook of Clinical Chemistry and Molecular Diagnostics, pp. 669-707, Elsevier Publishers, Philadelphia, Pa, USA, 5th edition, 2013.

[13] H.-X. Guo, C.-H. Wang, Z.-Q. Li et al., "The application of serum cystatin $\mathrm{C}$ in estimating the renal function in women with preeclampsia," Reproductive Sciences, vol. 19, no. 7, pp. 712717, 2012.

[14] Y. Fujita, I. Mori, and S. Kitano, "Color Reaction Between Pyrogallol Red - Molybdenum (VI) Complex and Protein," Japan Soc Anal Chem, vol. 32, no. 12, pp. E379-E386, 1983.

[15] N. Franceschini, C. Qiu, D. A. Barrow, and M. A. Williams, "Cystatin C and preeclampsia: a case control study," Renal Failure, vol. 30, no. 1, pp. 89-95, 2008.

[16] R. A. Lafayette, M. Druzin, R. Sibley et al., "Nature of glomerular dysfunction in pre-eclampsia," Kidney International, vol. 54, no. 4, pp. 1240-1249, 1998.

[17] S. J. VD Garovic, S. T. Wagner Turner et al., "Urinary podocyte excretion as a marker for preeclampsia," American Journal of Obstetrics \& Gynecology, vol. 196, no. 4, pp. 1-7, 2007.

[18] H. Strevens, D. Wide-Swensson, and A. Grubb, "Serum cystatin $\mathrm{C}$ is a better marker for preeclampsia than serum creatinine or serum urate," Scandinavian Journal of Clinical and Laboratory Investigation, vol. 61, no. 7, pp. 575-580, 2001.

[19] K. Kristensen, D. Wide-Swensson, C. Schmidt et al., "Cystatin C, $\beta$-2-microglobulin and $\beta$-trace protein in pre-eclampsia," Acta Obstetricia et Gynecologica Scandinavica, vol. 86, no. 8, pp. 921-926, 2007.

[20] G. A. Dekker and B. M. Sibai, "Etiology and pathogenesis of preeclampsia: Current concepts," American Journal of Obstetrics and Gynecology, vol. 179, no. 5, pp. 1359-1375, 1998.

[21] A. Conde-Agudelo and J. M. Belizán, "Risk factors for preeclampsia in a large cohort of Latin American and Caribbean women," British Journal of Obstetrics and Gynaecology, vol. 107, no. 1, pp. 75-83, 2000.

[22] A. L. Tranquilli, "Prediction, medical illness and the risk of preeclampsia," Pregnancy Hypertension, vol. 4, no. 3, p. 245, 2014.

[23] F. F. Kaze, F. A. Njukeng, A.-P. Kengne et al., "Post-partum trend in blood pressure levels, renal function and proteinuria in women with severe preeclampsia and eclampsia in Sub-Saharan Africa: a 6-months cohort study," BMC Pregnancy and Childbirth, vol. 14, no. 1, article 134, 2014.

[24] L. M. Amaral, M. W. Cunningham, D. C. Cornelius, and B. LaMarca, "Preeclampsia: long-term consequences for vascular health," Vascular Health and Risk Management, vol. 11, pp. 403415, 2015.

[25] A. O. Grubb, "Cystatin C-Properties and use as diagnostic marker," Advances in Clinical Chemistry, vol. 35, pp. 63-99, 2000.

[26] Y. Padma, V. B. Aparna, B. Kalpana et al., "Renal markers in normal and hypertensive disorders of pregnancy in Indian women: a pilot study," International Journal of Reproduction, 
Contraception, Obstetrics and Gynecology, vol. 2, no. 4, pp. 514520, 2013.

[27] K. M. Isasari, W. T. Pangemanan, I. Zulqarnain, and K. Rahadiyanto, "Maternal Cystatin C serum is higher in women with severe Preeclampsia," Indones J Obs Gynecol, vol. 2, no. 1, pp. 22-27, 2014.

[28] S. Back, E. Krutzen, and P. Nilsson-Ehle, "Contrast media as markers for glomerular filtration: a pharmacokinetic comparison of four agents," Scandinavian Journal of Clinical and Laboratory Investigation, vol. 48, no. 3, pp. 247-253, 2009.

[29] L. Cataldi, M. Mussap, L. Bertelli, N. Ruzzante, V. Fanos, and M. Plebani, "Cystatin C in healthy women at term pregnancy and in their infant newborns: Relationship between maternal and neonatal serum levels and reference values," American Journal of Perinatology, vol. 16, no. 6, pp. 287-295, 1999.

[30] E. L. Knight, J. C. Verhave, D. Spiegelman et al., "Factors influencing serum cystatin $\mathrm{Clevels}$ other than renal function and the impact on renal function measurement," Kidney International, vol. 65, no. 4, pp. 1416-1421, 2004.

[31] R. Kumaresan and P. Giri, "A comparison of serum cystatin C and creatinine with glomerular filtration rate in indian patients with chronic kidney disease," Oman Medical Journal, vol. 26, no. 6, pp. 421-425, 2011.

[32] A. N. Mikic, V. Cabarkapa, A. Nikolic et al., "Cystatin C in preeclampsia," Journal of Maternal-Fetal and Neonatal Medicine, vol. 25, no. 7, pp. 961-965, 2012.

[33] C. W. Redman, L. J. Beilin, and J. Bonnar, "Renal function in preeclampsia," Journal of Clinical Pathology, vol. 29, supplement 10, pp. 91-94, 1976.

[34] C. W. G. Redman, L. J. Beilin, J. Bonnar, and R. H. Wilkinson, "Plasma-urate measurements in predicting fetal death in hypertensive pregnancy," The Lancet, vol. 1, no. 7974, pp. 1370-1373, 1976.

[35] E. Schuster and B. Weppelmann, "Plasma urate measurements and fetal outcome in preeclampsia," Gynecologic and Obstetric Investigation, vol. 12, no. 3, pp. 162-167, 1981.

[36] N. Sagen, K. Haram, and S. T. Nilsen, "Serum Urate as a Predictor of Fetal Outcome in Severe Pre-Eclampsia," Acta Obstetricia et Gynecologica Scandinavica, vol. 63, no. 1, pp. 7175, 1984.

[37] K. P. Conrad and M. D. Lindheimer, "Renal and cardiovascular alterations," in Hypertensive disorders in pregnancy Stamford (CT), pp. 263-326, Appleton and Lange, 1999.

[38] O. Shemesh, H. Golbetz, J. P. Kriss, and B. D. Myers, "Limitations of creatinine as a filtration marker in glomerulopathic patients," Kidney International, vol. 28, no. 5, pp. 830-838, 1985.

[39] A. S. Levey, R. D. Perrone, and N. E. Madias, "Serum creatinine and renal function," Annual Review of Medicine, vol. 39, pp. 465490, 1988.

[40] R. D. Perrone, N. E. Madias, and A. S. Levey, "Serum creatinine as an index of renal function: new insights into old concepts," Clinical Chemistry, vol. 38, no. 10, pp. 1933-1953, 1992.

[41] H. Strevens, D. Wide-Swensson, and I. Ingemarsson, "Blood pressure during pregnancy in a Swedish population; impact of parity," Acta Obstetricia et Gynecologica Scandinavica, vol. 80, no. 9, pp. 824-829, 2001.

[42] K. Haram, E. Svendsen, and U. Abildgaard, "The HELLP syndrome: clinical issues and management. A Review," $B M C$ Pregnancy and Childbirth, vol. 9, 8 pages, 2009.

[43] H. Strevens, D. Wide-Swensson, O. Torffvit, and A. Grubb, "Serum cystatin $\mathrm{C}$ for assessment of glomerular filtration rate in pregnant and non-pregnant women. Indications of altered filtration process in pregnancy," Scandinavian Journal of Clinical and Laboratory Investigation, vol. 62, no. 2, pp. 141-147, 2002. 


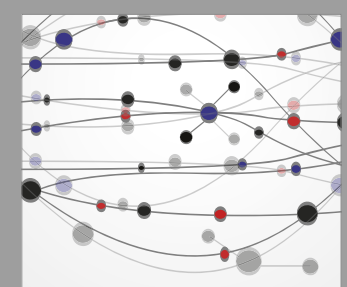

The Scientific World Journal
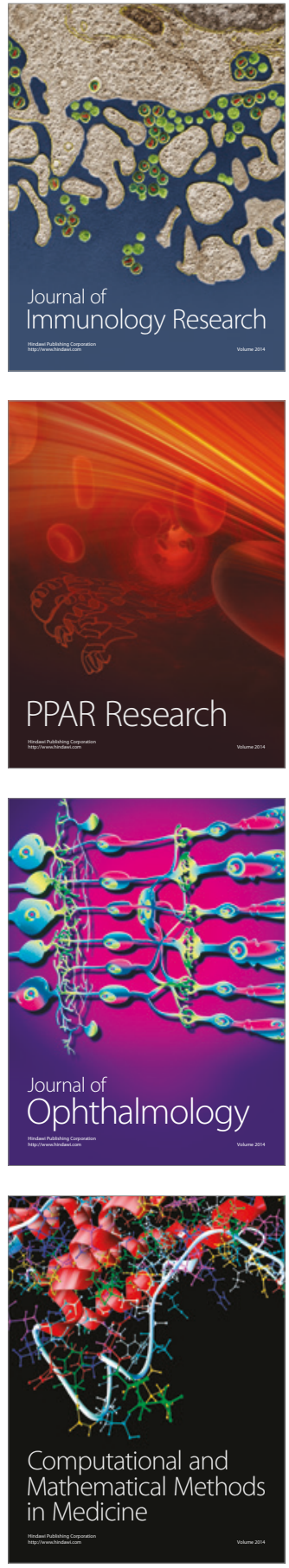

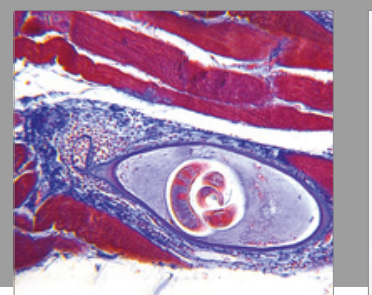

Gastroenterology Research and Practice
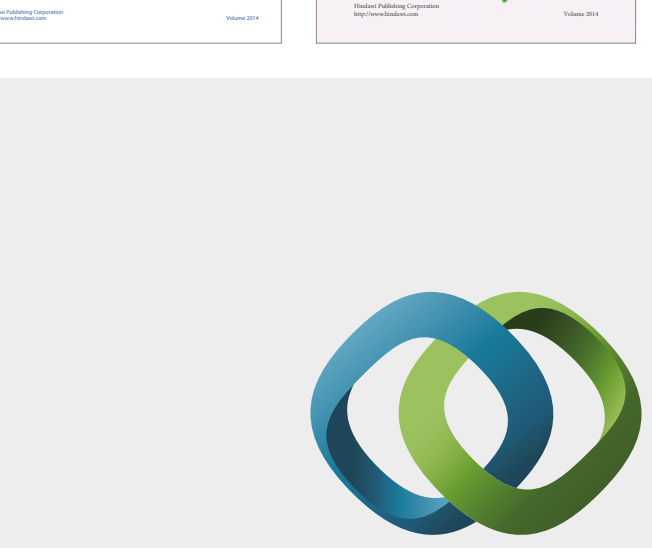

\section{Hindawi}

Submit your manuscripts at

https://www.hindawi.com
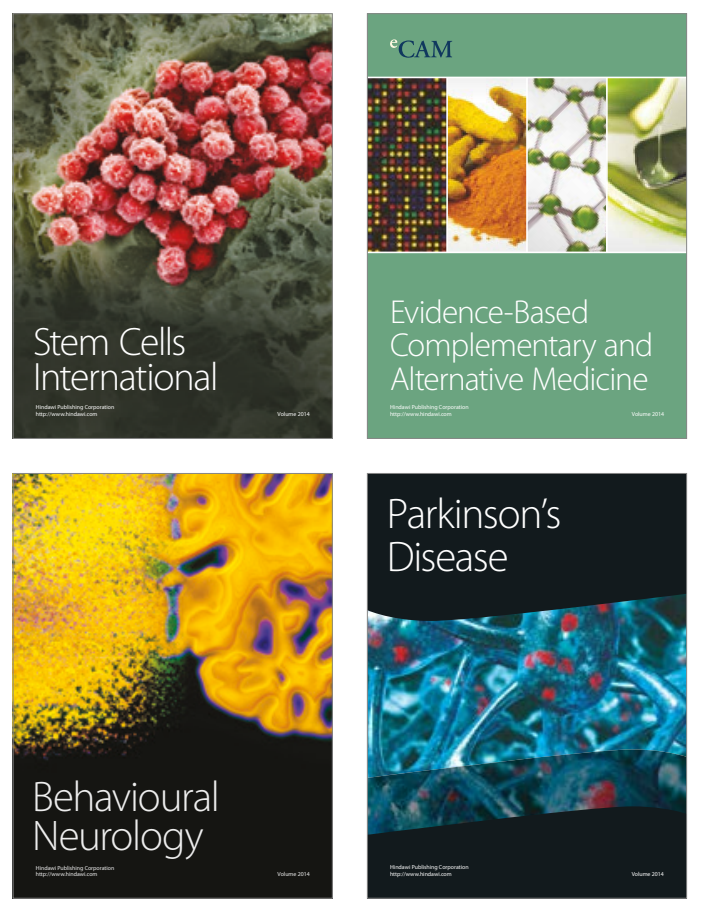
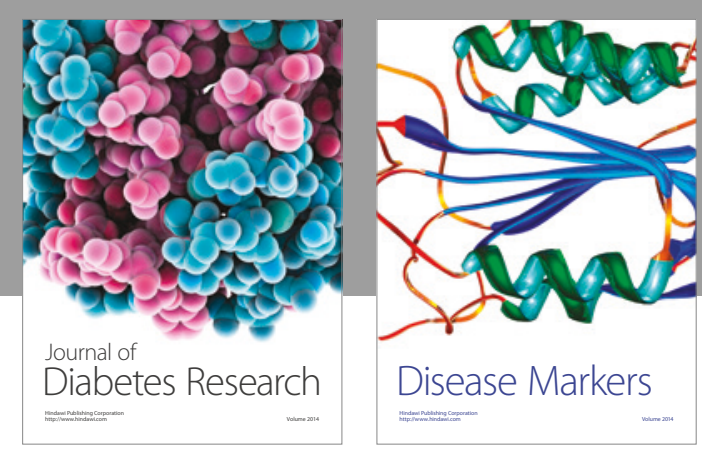

Disease Markers
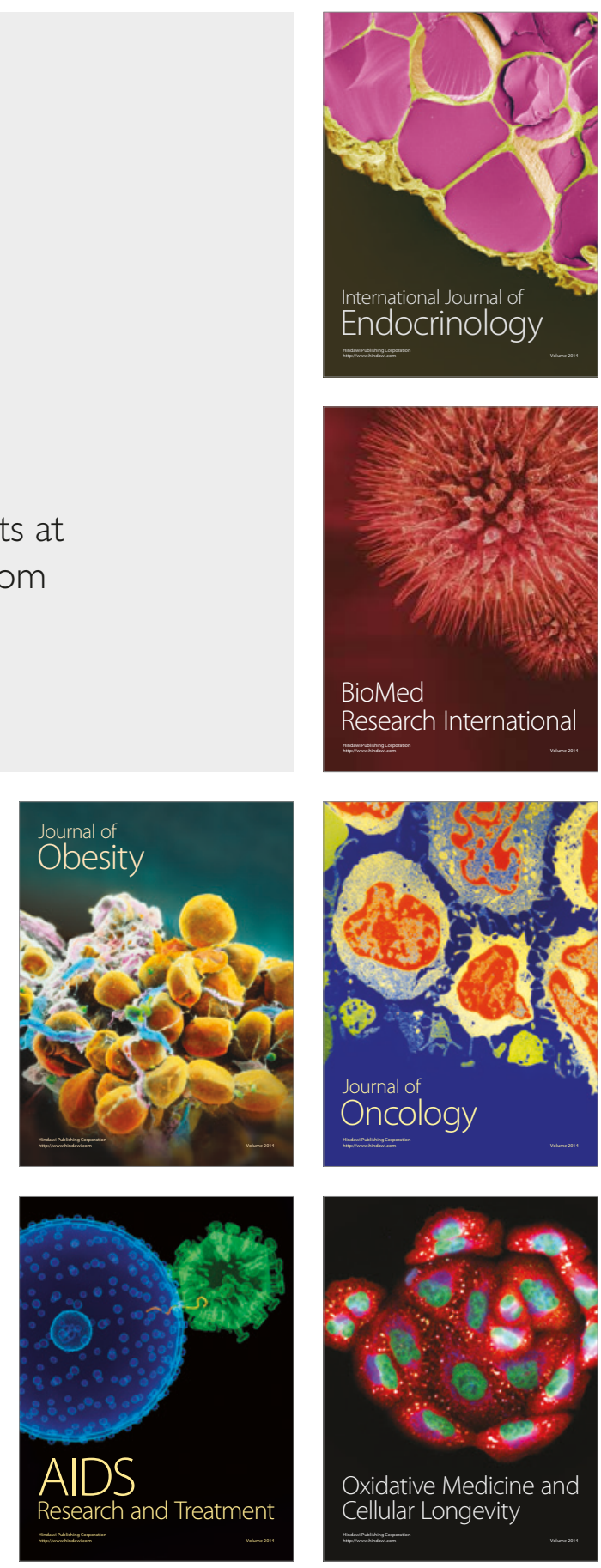